\title{
Power Saving in Multimedia Streaming Services in Cloud Computing
}

\author{
Sanjay J.Misal ${ }^{1}$, Dr.S. N. Mali ${ }^{2}$ \\ PG Student, Dept. of Electronics and Telecommunication, SITS, Narhe, Pune, India ${ }^{1}$ \\ Principal, Sinhgad Institute of Technology and Science, Narhe, Pune, India ${ }^{2}$
}

\begin{abstract}
In multimedia device power saving control mechanism improves lifetime of device. There are various techniques to reduce power consumption in multimedia devices. Such Systems mainly designed by using ARM controller which supports different features and algorithms for the development of streaming multimedia systems. To enhance the efficiency in developing embedded systems and to reduce burning kernel time, ARM controller is mounted by network file system (NFS) sharing multimedia file. DVFS gives good power control mechanism use different frequencies at different device state. ARM controller is powerful processor and it can be mounted on Linux, android operating systems which give highly effective and low power system.
\end{abstract}

Keywords: Multimedia, DVFS, Cloud services.

\section{INTRODUCTION}

There are open source and licence free software to develop multimedia devices. It gives application framework that can be add and modify functions, applications. Today's worlds these systems are used in home appliances, vehicles and smartphones to provide different services. A complete software frameworks includes Operating system, middleware and applications. The many systems based on Linux kernel. The uppermost layer is to develop applications and it can give to the third party. Many of the libraries are based on $\mathrm{C} / \mathrm{C}++$. In most of the mobile devices power is main concerns because of the overloading of the services. Android uses the Linux kernel power management technique to extend the battery life.

Multimedia devices increases day by day and also becoming more popular .They are using with device like mobile, laptop, smart phone and other embedded ones. Multimedia services are used in different fields like broadband, business, agricultural and industrial [1]. Streaming services are those which playing on one device and same time saving it on another. The directed media player will access file and play it without moving or copying[2].

\section{AN OVERVIEW OF DEVICE TECHNOLOGIES}

\section{A. Cloud Technology}

Cloud also capable to provide storage and multimedia content management with taking care of privacy and security. Cloud media system is layered like structure.

i)Infrastructure-as-a-Service :Resources are taken out from architecture or infrastructure. Ex. private, public clouds and community clouds.

ii)Platform-as-a-Service: Services in this type categorized as Media representation, Media Distribution and media adoption.

iii)Software-as-Service: In this services application are used by viewers itself on the device[3].
Mobile Cloud Computing system is helpful to remove drawback of system regarding the data storage and processing power. Mobile cloud computing also support security and mobility functions. Mobile cloud computing is consist of both technologies that is cloud computing and mobile computing [4]. Scalable coding-While playing multimedia services need to decode first so this decoding can be control to reduce power consumption. Feedback mechanism is used to give acknowledge to server and server changes data accordingly. When mobile device reduces its battery .RTSP is used for manage sequence which is helpful in controlling multimedia and playback[45-6].

\section{B. Platform and Standard}

System is mostly platform dominant .In this there is must requirement that developer do slightly different application version to address variation in sets. This may require dozens of executable files called as fragmentation that requires much of operational cost. Dot.NET CF Designed for application on windows mobile. It have ability with underlying devices operating system that allow integration of native component it the device[7].

3GPP is standard for multimedia it gives media codes and protocols for server and client.3GPP PSSS appeared first and its protocol were based on Real Time Steaming Protocol(RTSP), Request for Comments (RFC), Session Description Protocol for carrying real-time speech, audio, video. SMIL used for static media file and to carry continues media RTP/UDP/IP protocol was used [8].

\section{Android Technology}

Android launched in 2007 by Google. It is free and license free platform for developing mobile devices. Android have upper application layer and framework helpful to modify and add functions and applications. Today's worldandroid systems used many fields like home appliances, vehicles, smartphones, business and many others. 
III.

\section{AN OVERVIEW OF POWER AWARE MULTIMEDIA SYSTEM}

For reduce energy of system need to reduce the data rate of the multimedia stream. System which is designed by using ARM micro controller which supports different features and algorithms for the development of streaming multimedia.

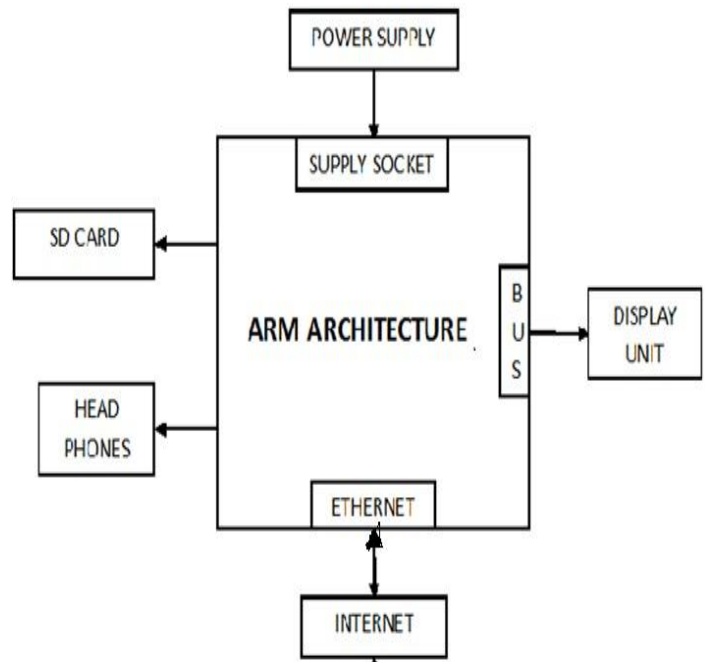

Fig 1.General architecture of Multimedia system

Integrated circuit technologies consume less power. ARM controller is a highly effective and low power hardware processor. It can be mounted on Linux, Android, and WINCE operating systems. The inbuilt DSP is a very powerful and very fast microprocessor. The analog to digital converter (ADC) can translate analog signals to digital signals. The DSP can translate digital signals to analog signals by digital to analog converter (DAC), which is useful for developing codec engines. To enhance the efficiency in developing embedded systems and to reduce burning kernel time, ARM controller is mounted by network file system (NFS) sharing multimedia file. It uses Transmission

Control Protocol/ Internet Protocol (TCP/IP) to mount on local network server. Through remote control, the user can add, modify, and remove applications and files. ARM board also support MMC multimedia cards for storing data.

The key element of an ARM RISC processor that the reduced instruction set means that the processor can run on using fewer transistors and hence reduce current consumption. The current consumption of any processor is a key attribute for many portable applications because it directly reflects into battery life.

\section{A. SYSTEM WITH CAMERA CAPTUTR UNIT}

Portable, battery operated embedded systems have more demands in terms of high performance and low microprocessor design. Dynamic voltage and frequency scaling is known technology to reduce energy consumption. This designed architecture is also support DVFS mechanism. It may hamper the slow voltage transitions. The recent technique like multithreading, task scheduling motivates the need of DVFS mechanism per core. Voltage regulators fielded into the same chip provide control and scaling voltage per core. The system may leads challenges like regulator efficiency transient characteristics etc. but on chip regulators are helpful to improve the effectiveness of DVFS and leads to energy saving of overall system.

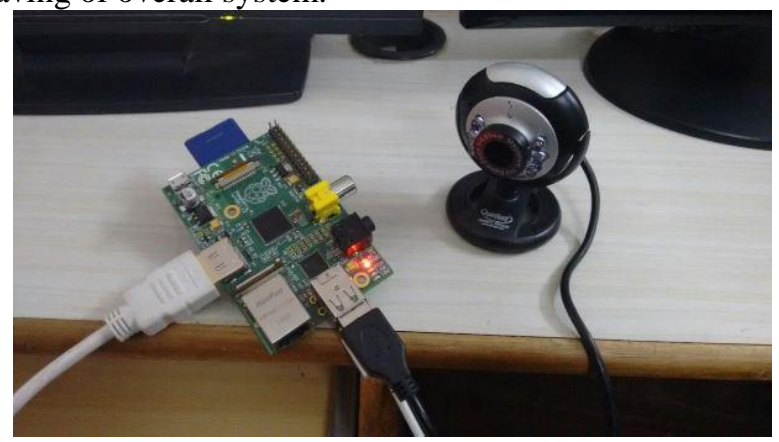

Fig 2.System with Camera Unit

The camera capture attached with system is for streaming the videos. The video can be streamed from the camera unit to the cloud server or it can be streamed from YouTube means through internet. While playing video hardware support DVFS mechanism that is it changes the cpu speed and voltage according to the device load. Which will helpful to save power of the processor.

\section{B. SERVER SIDEVIEW}

Cloud is implemented on local server. On this cloud we uploaded the video files of different lenths. The energy of offloading can be calculated, So energy is calculated with the help multiplication of of file length and time period required to offload. We can upload any content length file to the cloud only taking capacity into mind.
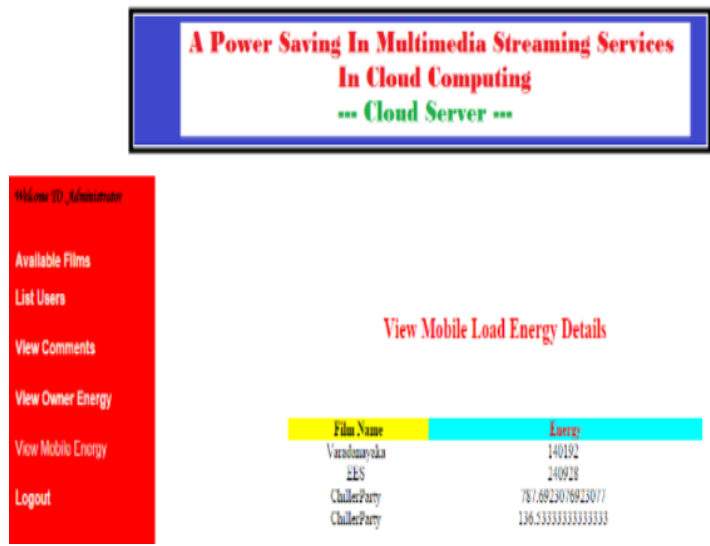

View Nobile Load Energy Detaik

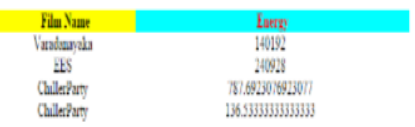

Fig 3. Server Side

So on the server side we can view all the files and energy details of file which is uploaded on the Server side.It is helpful to see all owner's files and viewing energy details.Mobile energy also can be calculated. Here owner can upload the files to the cloud server. To access the files from cloud to the user it need to login from client side.

\section{CLIENT SIDE VIEW}

Client side it is the side of the user. At the user end it is Gui for login and accessing the files. User can 
simplyaccess the videos, images, Audio files by simply login to it. Login keys are necessary for security purposes. The client GUI created here is on android operating system. This android application is mounted on development board. So it is possible to use boards low power technics and minimize power consumption, It is better and user friendly to use android system because now days it is very popular gives quality experience.

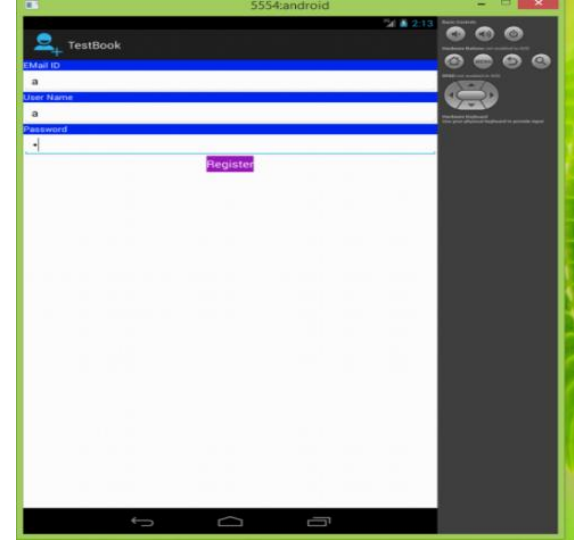

Fig 4.GuiFor Login

GUI shown above is android gui and user can register here by using the id and password and then further can login and access the file from the cloud.

\section{EXPERIMENTATION AND RESULTS:}

A.

\section{Viewing Energy Details:}

Table: 1 Energy Details at server side

\begin{tabular}{cc}
\hline Film Name & Energy \\
\hline EES & 1230393 \\
Varadanayaka & 7826000 \\
ChillerParty & 120928 \\
ChillerParty.mp4 & 9417022.13592233 \\
ChillerParty.mp4 & $1.3107476756756756 \mathrm{E} 7$ \\
EES.mp4 & $1.8935299948586117 \mathrm{E} 7$ \\
ChillerParty.mp4 & 8082944.0 \\
Varadanayaka.mp4 & 7899323.564356436 \\
ChillerParty.mp4 & 6553738.378378378 \\
ChillerParty.mp4 & $1.4476914626865672 \mathrm{E} 7$ \\
ChillerParty.mp4 & 7238457.313432836 \\
ChillerParty.mp4 & 5242990.702702703 \\
EES.mp4 & $2.1288530867052022 \mathrm{E} 7$ \\
EES.mp4 & 6845568.475836432 \\
EES.mp4 & 8525268.148148147
\end{tabular}

In the above table or webpage gives the energy details of the files at server side. The table file shows that energy consumption for different files is different and it can be changes accordingly with time period. It gives better comparison of energy details of uploaded files.

A.

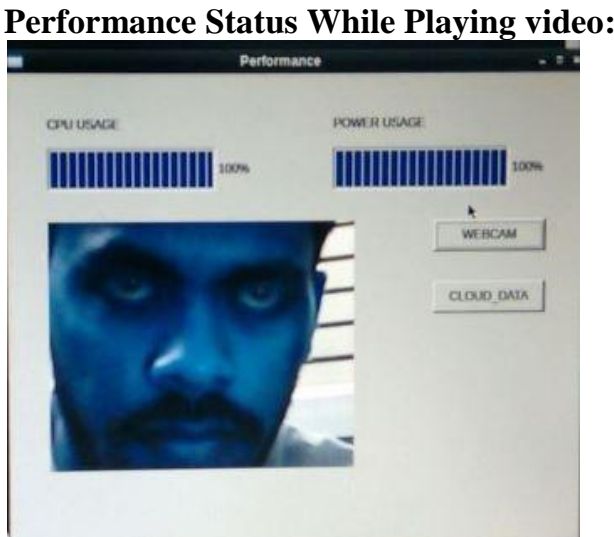

Fig 5. Performance WithCloud
In the above GUI the performance of the system is shown. This is screen on the client side which can observe the performance and it helpful to user to offloading the complex file into the cloud server. Which will improves the lifetime of the system. Here we monitored the parameter like CPU usage and power usage and can be add quality current also.

The GUI shows that while playing the video or accessing the cloud data the CPU used completely and and power used is also high. It can be change according to video load.

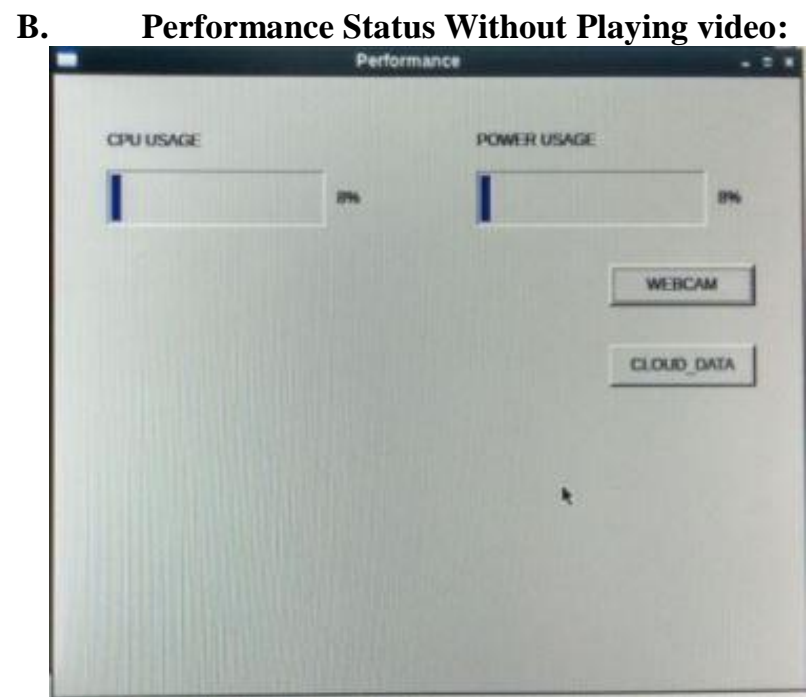

Fig 6. Performance Without Cloud

In the above GUI the performance of the system is shown without playing video files. The GUI shows that without playing the video or accessing the cloud data the CPU not used completely but it is only $10 \%$ and power used is also low. It can be increases according according to video load.

\section{CONCLUSION}

This different technologies and different mechanism used in multimedia devices to reduce power consumption. Android has open source and mainly used in multimedia devices to give more powerful system. In every mobile platform it is necessary to develop application framework and import with kernel. Then system can easily use the power management schemes of kernel. This system gives an approach that minimizes the energy consumption incurred by the backlight when users access multimedia streaming on mobile devices. Specifically, the approach exploits backlight scaling and models a fundamental optimization problem with scaling constraints (to limit image distortion, reflect hardware limitation, and consider user perception). To solve the problem, we propose three algorithms, and prove that they are optimal in terms of energy savings when the energy consumption increases strictly with the backlight levels.

\section{REFERENCES}

[1]. Yi-Wei Ma, Jiann-Liang Chen, Ching-Hesign Chou, and ShyueKung Luences, "A PowerSaving Mechanism For Multimedia 


\section{International Journal of Advanced Research in Computer and Communication Engineering}

Vol. 4, Issue 5, May 2015

Streaming Services in Cloud Computing” IEEE TRANSACTIONS ON MULTIMEDIA, VOL. 8, NO. 1, MARCH 2014

[2]. Giovanni Schembra and Giuseppe Incarbone "A Business Model for Multimedia Streaming In Mobile Clouds" IEEE transactions on network and service management, Vol. 11, no. 3, september 2014

[3]. Snehal P.Warhekar,.V.T.GaikwadSipna COET , "Implementing Mobile Multimedia Applications Using Cloud Computing" (IJCSIT) International Journal of Computer Science and Information technologies, vol. 5 (1) , 2014, 230-232

[4]. Yonggang wen, senior member, IEEE, xiaoqingzhu, member, IEEE, joel j. P. C. Rodrigues, Senior member, ieee, and chang wen chen, fellow, IEEE ,"Cloud mobile media:Reflections and outlook" IEEE transactions on multimedia, vol. 16,No. 4, june 014

[5]. Shitala prasad1, sateesh k. Peddoju2 and debashis ghosh3, "Agromobile: a cloud-based Framework for agriculturists on mobile platform" international journal of advanced science And technology vol.59, (2013), pp.41-52.

[6]. Duong QuocTrong, HeikoPerkuhn, and Daniel Catrein ,Dynamically Scaling Multimedia Streaming Service on Hybrid Telco Cloud" Proceedings of the World Congress on Engineering and Computer Science 2011 Vol I WCECS 2011, October 19-21, 2011, San Francisco, USA

[7]. DamianosGavalas and Daphne Economou , "Development Platforms for Mobile Applications: Status and Trends" IEEE SOFTWARE// WWW.COMPUTER.ORG/SOFTWARE

[8]. FrdricGabin, Markus Kampmann, Thorsten Lohmar, and Clinton Priddle , "3GPP Mobile Multimedia Streaming Standards" IEEE SIGNAL PROCESSING MAGAZINE [134] NOVEMBER 2010

[9]. Wonyoung Kim, Meeta S. Gupta, Gu-Yeon Wei and David Brooks , "System Level Analysis of Fast, Per-Core DVFS using On-Chip Switching Regulators" 978-1-4244-2070- 4/08/IEEE 2008

[10]. J. Adams and G.-M.Muntean, "Save adaptation algorithm for multimedia streaming to mobile devices," IEEE International Confer- ence on Portable Information Devices, Orlando, Florida, USA, 2007.

[11]. Adams and G.-M.Muntean,buer "Power save mechanism for mobile multimedia streaming," IEEE International Conference on Communications (ICC07), Glasgow, Scotland, UK, 2007.

[12]. L. Zhong and N. Jha, "Efficiency of handheld computer interfaces: limits, characterization and practice," Proceedings of the Third International Conference on Mobile Systems, Applications, and Services (MobiSys 2005), pp. 247 60, 2005.

[13]. [13] Video coding for low bit rate communication," tech. rep., International Telecommunication Union, 2005.

[14]. [14] Advanced video coding for generic audiovisual services," tech. rep., International Telecommunication Union, 2005. 